\title{
Superior External Articular Artery
}

National Cancer Institute

\section{Source}

National Cancer Institute. Superior External Articular Artery. NCI Thesaurus. Code C33673.

A blood vessel arising from the popliteal artery that divides into deep and superficial branches. The deep branch supplies blood to the lower portion of the femur and knee joint while the superficial branch supplies the vastus externus. 